\title{
Liver and intestine transplantation
}

\author{
John P. Roberts ${ }^{a}$, Robert S. Brown, Jr ${ }^{b}$, Erick \\ B. Edwards ${ }^{c}$, Douglas G. Farmerd, Richard \\ B. Freeman, $\mathrm{Jr}^{\mathrm{e}}$, Russell H. Wiesner ${ }^{\mathrm{f}}$ and Robert \\ M. Merion ${ }^{9 *}$
}

a University of California San Francisco, San Francisco, CA ${ }^{\mathrm{b}}$ Columbia University College of Physicians and Surgeons, New York, NY

'Organ Procurement and Transplantation Network (OPTN)/ United Network for Organ Sharing (UNOS), Richmond, VA

'University of California Los Angeles, Los Angeles, CA

e Tufts-New England Medical Center, Boston, MA

${ }^{\dagger}$ Mayo Clinic, Rochester, MN

${ }^{9}$ Scientific Registry of Transplant Recipients (SRTR)/

University of Michigan, Ann Arbor, MI

*Corresponding author: Robert M. Merion, merionb@ umich.edu

Key words: Deceased donors, graft survival, intestine transplantation, liver transplantation, liver-intestine transplantation, living donors, MELD, organ donation, patient survival, PELD, SRTR, waiting list

Received 17 September 2002, revised and accepted for publication 12 December 2002

\section{Introduction}

Over the last several years, the general trend in organ transplantation has been a continued increase in the number of patients waiting for transplantation that has outpaced the much slower increase in the number of donor organs available. As evidenced by the 2001 data regarding liver transplantation, this shortage has led to a rapid increase in living donor transplantation. The doubling of the numbers of living donor transplantations in the last 2 years has resulted in about $10 \%$ of the transplants being living donor transplants. Dependent on the acceptance of

Funding: The Scientific Registry of Transplant Recipients (SRTR) is funded by contract \#231-00-0116 from the Health Resources and Services Administration (HRSA). The views expressed herein are those of the authors and not necessarily those of the US Government.

This is a US Government-sponsored work. There are no restrictions on its use.

Note on Sources: The articles in this supplement are based on the reference tables in the 2002 OPTN/SRTR Annual Report, which are not included in this publication. Many relevant data appear in figures and tables directly referred to in the article; other tables from the Annual Report that serve as the basis for this article include the following: Tables 1.1, 9.1-9.4, 9.7-9.9, 10.1-10.4, and 10.7-10.10. All of these tables are also available online at http:// www.ustransplant.org. the risk to the donor, living donor liver transplantation is expected to reach as high as $15 \%$ of the liver transplants performed in the United States in the future (1).

Another change, the effect of which is only beginning to be seen in the data, has been the institution of the Model for End-stage Liver Disease (MELD) for prioritization of adult liver transplant candidates and the PELD system for pediatric candidates. This system was instituted in February 2002. In this report the authors present some preliminary results for this system and some data comparing the MELD/PELD system with the previous allocation plan. This report will first summarize data from the previous decade, the vast majority of which was derived prior to implementation of the MELD/PELD system. Preliminary MELD/PELD results will be presented in the final section of the report on liver transplantation.

Intestinal transplantation is fraught with a higher risk of death for patients awaiting transplantation than for patients awaiting liver transplantation, despite advances in surgical technique, patient selection, and immunosuppressive protocols.

Unless otherwise noted, the statistics in the following article come from reference tables in the 2002 OPTN/ SRTR Annual Report. Two companion articles in this report, 'Data Sources and Structure' and 'Analytical Approaches for Transplant Research', explain the methods of the data collection, organization, and analysis that serve as a basis for this article $(2,3)$.

\section{Liver Transplantation}

\section{Liver waiting list characteristics}

Over the past decade, the number of patients waiting for liver transplantation increased nearly ninefold, from 2217 in 1992 to a peak of 18505 in 2001 (Figure 1). During this period, the number of cadaveric liver transplants performed rose from 3031 in 1992 to 4665 in 2001, while the number of living donor liver transplants increased much more rapidly_from 33 in 1992 to 515 in 2001. Between 1992 and 2001, the number of patients older than 50 listed for liver transplantation rose from 891 patients $(40 \%)$ to 10629 (57\%). Although the number of children under 5 years of age listed for liver transplantation increased from 205 to 543 during that same period, their percentage of the waiting list decreased from $9 \%$ to $3 \%$. There also has been an increase in the percentage of waitlisted adult males, from $50 \%$ in 1992 to $57 \%$ in 2001 . The ethnic composition of the waiting list has changed very little over the past decade, with whites making up 


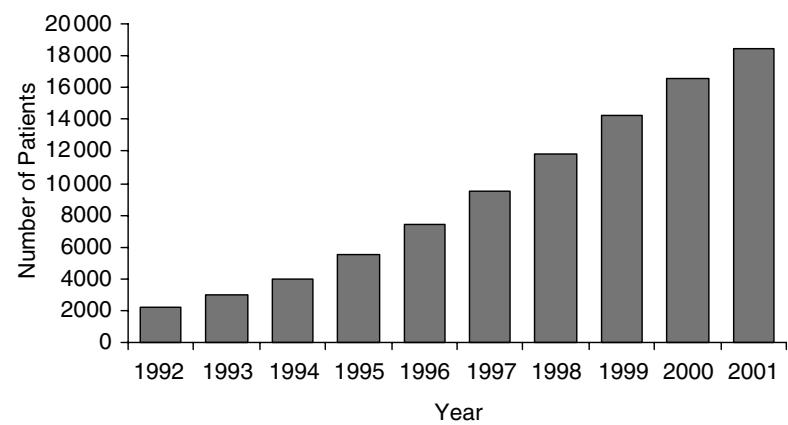

Figure 1: Registrants on the liver waiting list, 1992-2001. Source: 2002 OPTN/SRTR Annual Report, Table 9.1.

approximately $86 \%$ of the list, African Americans 8\%, and Asians 4\%. Similarly, the composition of the waiting list with respect to blood type has changed very little, with approximately $50 \%$ of the waiting list being blood type $\mathrm{O}$, $35 \%$ blood type A, 12\% blood type B, and 3\% blood type $A B$. Finally, the percentage of patients listed for retransplantation decreased slightly from $10 \%$ in 1992 to $7 \%$ in 2001 . This shift may be attributable to a lower incidence of primary nonfunction or a reduced propensity to relist patients with recurrent hepatitis $\mathrm{C}$.

As might be expected, waiting time increased dramatically over the decade: The percentage of candidates on the list who had been waiting 2 years or longer for a liver rose from $13 \%$ at the end of 1992 to nearly $40 \%$ at the end of 2001 (Figure 2). While the absolute number of annual deaths on the liver transplant waiting list has increased (Figure 3), the annual death rate per 1000 patient years at risk on the waiting list has decreased from 270 in 1992 to 115 in 2001 . The annual death rate per 1000 patient years at risk for patients listed at each medical urgency status level is noted in Figure 4 for 2001. Patients listed at Status 2A were at highest risk of dying. It must be noted, however, that listing practices have changed during the past decade, and in recent years many patients appear to

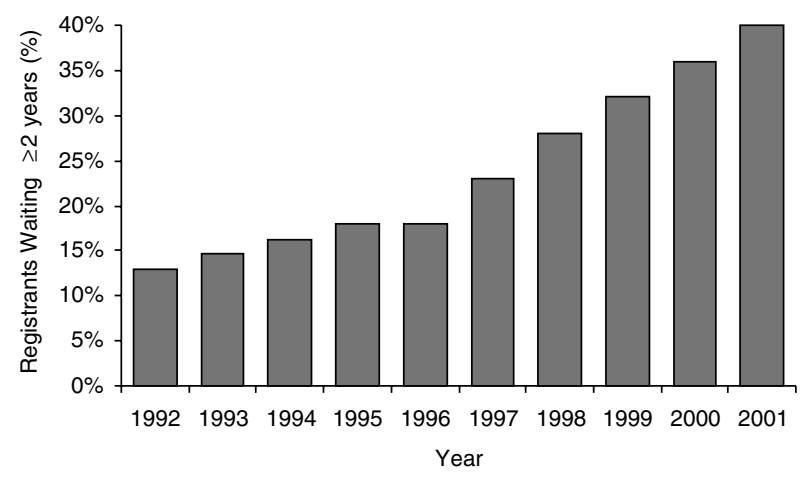

Figure 2: Registrants waiting 2 years or more for a liver transplant. Source: 2002 OPTN/SRTR Annual Report, Table 9.1.

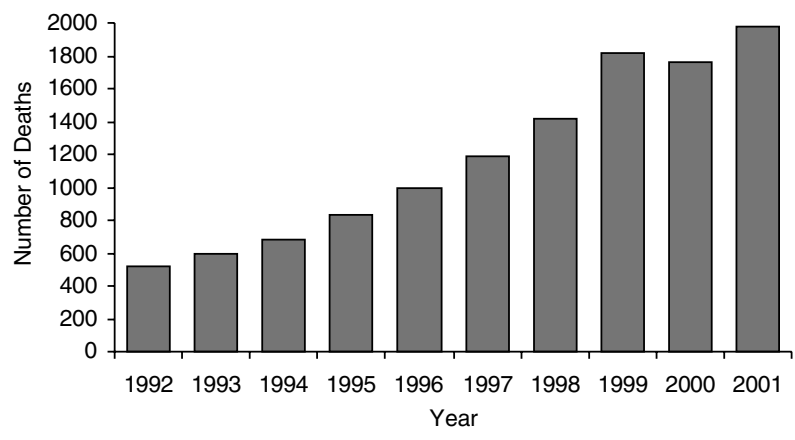

Figure 3: Deaths on the liver waiting list, 1992-2001. Source: 2002 OPTN/SRTR Annual Report, Table 9.3.

have been added to the liver waiting list earlier in the course of their disease, with the expectation of a progressively longer waiting time. Therefore, the death rate on the waiting list may be less meaningful for recent years. This is supported by the fact that the percentages of patients listed as Status 1, 2A, and 2B have been quite stable in recent years. In general, Status 1 patients made up $0.1 \%$ of the list, Status 2A 0.5\%, Status 2B 17\%, and Status 3 $66 \%$, with approximately $15 \%$ of patients being listed as inactive. These data support the concept that waiting time is not a good predictor of mortality on the waiting list (4). The inability of waiting time to predict wait list mortality was one of the primary driving forces behind the implementation of the MELD and PELD systems (see below) (5).

\section{Trends in liver waiting list mortality}

Despite the growing disparity between the number of deceased donors and patients waiting for a liver transplant, overall waiting list death rates dropped from 270 per 1000 patient years at risk in 1992 to 115 in 2001 (Figure 5). This decrease in death rates may be attributed in part to increasing numbers of less medically urgent patients on the waiting list. At the end of 1992, 13\% of patients on the liver waiting list had been waiting 2 or more years for a transplant; at the end of 2001, the

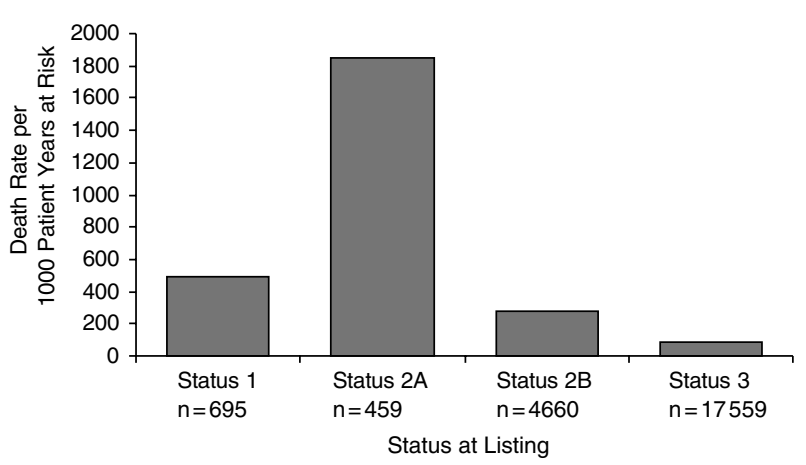

Figure 4: Death rate per 1000 patient years at risk, by status at listing, 2001. Source: 2002 OPTN/SRTR Annual Report, Table 9.3. 
Roberts et al.

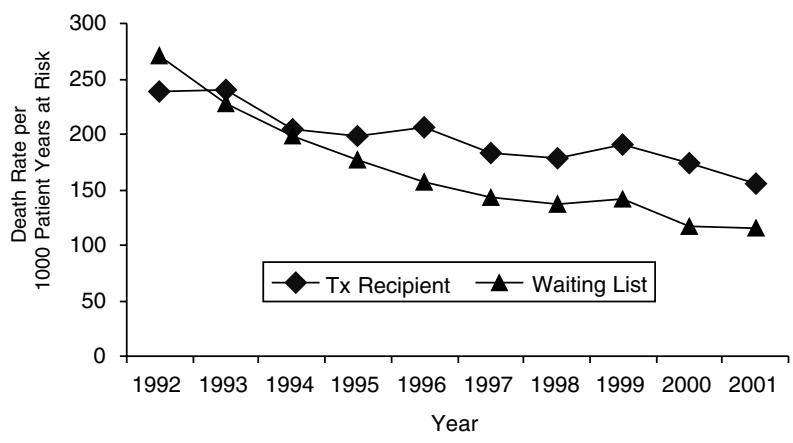

Figure 5: Death rates per 1000 patient years at risk, liver transplant recipients vs. liver waiting list, 1992-2001. Source: 2002 OPTN/SRTR Annual Report, Tables 9.3, 9.7.

percentage of patients with this lengthy wait was nearly $40 \%$ - a shift suggesting more candidates with less medically urgent need. However, death rates have steadily declined since 1992 for each medical urgency status, indicating that improvements in pretransplant patient care, as well as changes in case mix, also may be responsible for the trend toward lower death rates.

\section{Liver transplant recipient trends}

In interpreting trends in the distribution of medical urgency status, it should be noted that definitions were not uniform during the period of this report. In 1997, OPTN/ UNOS approved several key changes to the definitions of medical urgency status. The least urgent classification, Status 4, was eliminated. At the time of the change, existing Status 4 patients were 'grandfathered' into Status 3. This change explains the large increase in the percentage of Status 3 registrations on the waiting list at the end of 1997. The most medically urgent classification, Status 1 , was redefined to include patients without a previous history of liver disease (acute fulminant hepatic failure), patients with primary nonfunction of a transplanted liver, patients with hepatic artery thrombosis, and certain pediatric patients. Status $2 \mathrm{~A}$ was created for patients with a history of liver disease who critically decompensate. In 1998, the classifications for Status 2A, Status 2B, and Status 3 underwent further refinement as the Child-TurcottePugh scoring system was selected to serve as the basis for categorizing patients with chronic liver disease. Thus, at the end of 2001 , nearly $82 \%$ of patients waiting were classified as Status 2B or Status 3. Similarly, about $82 \%$ of patients added to the waiting list during that year were designated either Status 2B or Status 3. In 2002, the existing medical urgency status system for patients with chronic liver disease was terminated in favor of MELD. Status 1 was retained for patients with acute disease.

Since 1998, the percentage of Status 1 and Status 2B recipients has remained relatively unchanged. Status $2 A$ represented the fastest growing group of recipients, increasing from $17 \%$ in 1998 to $25 \%$ in 2001. In contrast, the percentage of Status 3 transplants decreased from $17 \%$ in 1998 to $9 \%$ in 2001. Notably, of the Status 2A recipients transplanted in 2001, more than $40 \%$ were initially listed in Status 3 and about 35\% were initially listed in Status 2B, indicating that, as the waiting times increase, a growing number of healthier patients will critically decompensate. It is hoped that the MELD system may represent an improvement in the ability to prioritize the patients listed for transplantation in order to allow transplantation of some of these patients with a high risk of decompensation (6).

The percentage of cadaveric liver transplants going to pediatric patients decreased from 15\% in 1992 to $10 \%$ in 2000. With few exceptions, pediatric patients received a smaller percentage of transplants during each year of the period. This may be due to the relentless rise in the number of adults listed and transplanted because of the hepatitis $\mathrm{C}$ epidemic. At the same time, there has not been a similar increase in the number of pediatric candidates listed or transplanted, resulting in a steady decrease in the proportions of pediatric candidates and recipients relative to adults. In 2001, the OPTN implemented a policy in which pediatric livers would be offered preferentially to pediatric candidates, anticipating a slight increase in the number of pediatric patients transplanted. During the implementation year, the percentage of cadaveric transplants going to pediatric recipients rose more than in any other year of the period (from $10.1 \%$ in 2000 to $10.5 \%$ in 2001).

\section{Living donor liver transplantation}

The number of living donor transplants increased markedly over the last 10 years, rising from 33 recipients in 1992 to 515 in 2001 (Figure 6). Most of this increase occurred between 1999 and 2001 because of the exponential growth of adult-to-adult living donor transplantation. During this same time frame, the number of pediatric living donor recipients increased only modestly (Figure 6). As a result, among all living donor liver recipients, the age distribution is bimodal, with $21 \%$ being pediatric recipients and a second peak at age 50-64 years. In 2001, 59\% of living donor recipientswere males-lower than the percentage of males receiving livers from deceased donors and slightly higher than the percentage of males on the liver waiting list. In the same year, $86 \%$ of living donor liver recipients were white. The percentage of African American recipients of living donor livers decreased steadily from 1998 (20\%) to 2001 (4\%)-lower than the percentage of African American recipients of livers from deceased donors $(9 \%)$ or African Americans on the liver waiting list (8\%). Whether 2001 represents an aberration or changing demographics among recipients of living donor liver transplantation remains to be seen. In one study, African American patients listed for living donation were less likely to have a donor than white patients of 


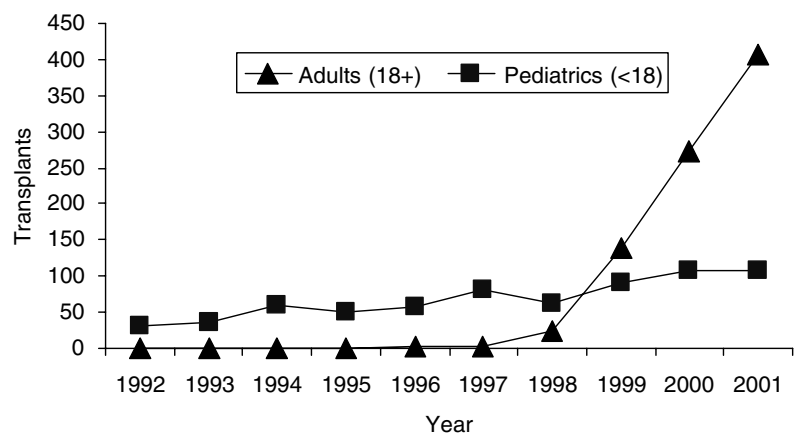

Figure 6: Living liver donor transplants, adults vs. pediatrics, 1992-2001. Source: 2002 OPTN/SRTR Annual Report, Table 9.4.

Hispanic or non-Hispanic ethnicity (7). Hispanic ethnicity was similar among living donor recipients, deceased donor liver recipients, and those on the cadaveric waiting list (ranging from $13 \%$ to $15 \%$ ). Nonresident aliens represented a higher proportion of living donor recipients (4\%) than deceased donor liver recipients $(2 \%)$ or those on the liver waiting list (1\%).

The diagnoses among recipients of living donor grafts also differ somewhat from their deceased donor counterparts. In 2001, fewer recipients with noncholestatic liver disease received transplants from living donors (55\% living, 61\% deceased); higher proportions were transplanted for cholestatic liver disease (16\% living, 10\% deceased) and malignant neoplasm (5\% living, 3\% deceased). Among patients undergoing living donor liver transplantation, the proportion performed for biliary atresia fell from $76 \%$ of the total in 1992 to $8 \%$ in 2001 , even though the absolute number rose from 25 to 44 . The percentage drop was precipitous in 1999, likely because of the increased adult living donor liver transplant activity, which overshadowed the activity in children at that time. In 2001 , only $2 \%$ of living donor liver recipients had a prior organ transplant of any type (and all were liver transplants); previous organ recipients made up $10 \%$ of deceased donor liver recipients and $8 \%$ of the liver waiting list.

Of the recipients of living donor transplants, only $4 \%$ of patients were on life support at time of transplant and $71 \%$ were not hospitalized-much lower than the corresponding percentages for deceased donor transplants (11\% on life support, $26 \%$ in the intensive care unit, and only $57 \%$ at home). Of the candidates on the waiting list, 16\% were Status 2B, 65\% were Status 3 (and likely at home), and fewer than $1 \%$ were in urgent categories. In 2001, the vast majority of living donor liver recipients were Status 2B (46\%) or Status $3(39 \%)$ at time of transplant. The number of living donor Status 1 transplants has decreased over the years, as very few adult patients (in contrast with pediatric patients), receive livers from living donors as Status 1. Recipients of livers from deceased donors were characterized by a higher level of urgency: only $9 \%$ at Status 3 and $54 \%$ at Status 2B, as well as $25 \%$ at Status $2 \mathrm{~A}$ and $13 \%$ at Status 1, the highest-urgency categories. In fact, some living donor recipients never receive a medical urgency status code at all, because not all are included on a waiting list before transplantation. Recent reports of living donor transplantation suggest that the results are better when recipients are less severely ill at the time of transplant $(8,9)$. Whether this has to do with the relative inexperience with this procedure or the small size of the liver graft as compared with cadaveric transplantation is unknown. An important data set is the demographics and outcomes of the living liver donors. The safety of this procedure has been scrutinized in the medical and lay press (10). Estimates of the mortality risk of liver donation for the adult-to-adult donation have been as high as $1 \%$. Future reports will contain data regarding the outcome of the donors and estimates of the risk of mortality.

\section{Liver transplantation death rates}

Annual death rates per 1000 patient years at risk for liver transplant recipients are calculated by assessing all of the liver recipients alive at the start of a given year, adding all of the recipients receiving a new transplant in that year, and determining the number of deaths for this combined group of patients. Each patient's survival time after transplant is determined. The number of recipients alive in a given year multiplied by the number of years each patient has been alive since transplant determines the number of patient years. This number serves as the denominator for the number of deaths occurring in that year. In 2001, overall annual death rates for liver recipients were 155 per 1000 patient years at risk, similar to the previous rates of 174 in 2000, 191 in 1999, and 178 in 1998 (Figure 5). It is interesting to note that death rates on the waiting list for the same time periods continued to decline. As in past years, pediatric recipients less than 1 year of age have the highest death rates, although only three such children died in 2001. Death rates for recipients of other age groups have fluctuated over the past decade, with the death rates for most age groups declining. When recipients are stratified by race, African Americans have higher death rates than whites or Asians. For 2 of the last 3 years, Hispanic/Latino recipients have exhibited consistently lower death rates. The trend toward higher death rates for African Americans and lower death rates for Hispanics has been noted previously (11). Male recipients had slightly higher death rates throughout much of the previous decade. Recipients with blood type $A B$ had the lowest death rate (121 deaths per 1000 patient years at risk) in 2001; blood types O, B, and A had successively higher mortality rates during the year $(147,160$, and 165 deaths per 1000 patient years at risk, respectively). Recipients with blood type $A B$ have had progressively lower death rates for each of the last 4 years. Interestingly, nonresident alien recipients had lower death rates than US resident recipients in 2001, but extremely few nonresident 


\section{Roberts et al.}

aliens received transplants. Liver recipients with previous transplants of any organ type had much higher death rates than first-time liver recipients in the 2001 cohort. Higher death rates for repeat transplant recipients have been evident for every preceding year in this decade.

Death rates for liver recipients are highest in the first year after transplantation and decline progressively as the interval from transplant becomes longer $(12,13)$. This reflects the increased risk of death around the transplant surgery and early postoperative period and the relatively few deaths that occur after the first year with a transplant. A relatively reproducible increase in death rate occurs for patients more than 5 years after their transplant, probably because comorbidities, recurrent primary liver disease, and drug toxicities accumulate at this stage $(12,13)$. Compared with patients called in from home, those receiving liver transplants while in the intensive care unit consistently had higher death rates in each year of the study period. Similarly, recipients maintained on life support at time of transplant had post-transplant death rates approximately twice as high over the same period. When recipients are stratified by primary liver disease diagnoses, those with malignancies had higher death rates than all other diagnoses, likely due to recurrent malignant disease (14). In 2001, recipients with noncholestatic cirrhosis, metabolic disorders, and cholestatic liver diseases had the lowest death rates. For the 2001 death rate analysis, the previous medical urgency status definitions segregated death rates well, with Status 1 recipients having the highest death rates, followed by Status $2 \mathrm{~A}$ and Status 2B. Previous patterns for higher death rates for recipients of livers from older donors were again observed in 2001. The outcome of recipients of the older donor liver is controversial $(15,16)$. A recent publication suggests that the outcome of patients with hepatitis $\mathrm{C}$ virus who are transplanted with older donor livers is impaired (17). Recipients of grafts from donors less than 1 year old had higher mortality rates, possibly an effect of technical issues prevalent with extremely small grafts and recipients. In the years 2000 and 2001, the death rates have decreased for recipients of living donor grafts, likely as a result of more experience and expertise with the living donor liver transplant evaluations and procedures.

\section{Liver transplantation graft survival}

Three-month and 1-year graft survival rates were calculated for patients receiving transplants in 1999 or 2000, 3 -year survival was calculated from recipients of grafts in 1997-1998, and 5-year graft survival was calculated from recipients transplanted in 1995-1996. Graft survival rates at 3 months and 1 year after transplantation of livers from deceased donors were highest for recipients in the middle age ranges, although the reduced graft survival for recipients at the extreme age ranges was less apparent in the 5-year graft survival cohort. Long-term graft survival was superior for Asian recipients, with whites and African
Americans having successively reduced graft survival rates, especially for the 5-year survival cohort. Women had slightly reduced early graft survival, but this difference was reversed for recipients transplanted in 1995 or 1996. Recipients receiving second grafts had consistently reduced graft survival at all time points. More ill recipients had poorer graft survival at all time points, regardless whether illness is defined by patient location (in hospital or ICU), need for life support, or OPTN/UNOS status code. Transplant center volume accounted for relatively little difference in graft survival, with the largest and smallest centers having slightly reduced graft survival rates compared with other centers. This parallels the results in deceased donor liver transplantation. However, these data were not adjusted for other confounding variables, including severity of recipient illness at time of transplantation. This may explain differences from earlier publications showing lower survival rates in lower-volume centers (18). Deceased donor grafts from the youngest and oldest donors had inferior survival rates for all time periods examined.

Graft survival for living and deceased donor livers for 3 months, 1 year, 3 years, and 5 years are shown in Figure 7. The rates of graft survival for living donor grafts are lower than for cadaveric grafts at 3 months and 1 year after transplant, but better after 5 years. This is perhaps because the majority of the living donor recipients in the 5-year survivor cohort are pediatric recipients. In fact, recipients younger than 1 year achieved the best living donor graft survival rates for each survival time interval except 5 years, where ages 1-5 were superior. The decline in graft survival rates as severity of liver disease increased, as observed for cadaveric grafts, was more pronounced for living donor grafts. In 2001, graft survival for living donor liver recipients was $76 \%$ at 1 year, compared with $80 \%$ for deceased donor liver recipients (Figure 7). Longer-term graft survival (more than 3 years) likely reflects predominantly pediatric recipients of living donor livers and is stable at $73 \%$.

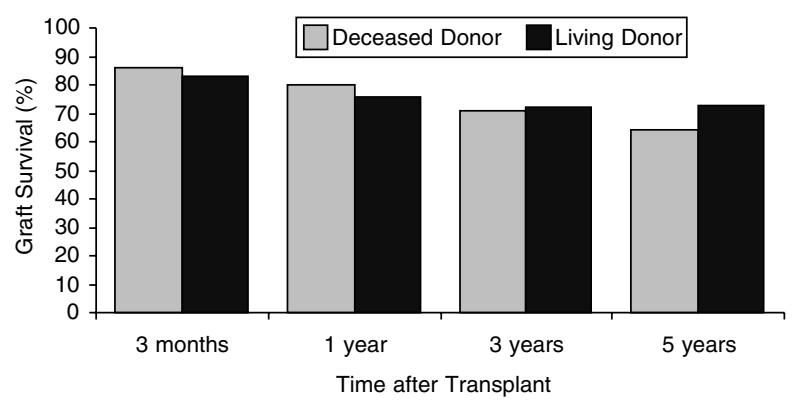

Figure 7: Graft survival among liver transplant recipients, living vs. deceased donor. Source: 2002 OPTN/SRTR Annual Report, Table 9.8. Cohorts are for transplants performed during 1999-2000 for 3-month and 1-year; 1997-1998 for 3-year; and 1995-1996 for 5 -year survival. 


\section{Liver transplantation patient survival}

Patient survival rates were calculated using survival cohorts similar to those used for the graft survival calculations. For patients receiving transplants in 1999 and 2000, recipients of deceased donor liver transplants had a 1-year survival rate above $86 \%$. Patient survival for living and deceased donor livers for 3 months, 1 year, 3 years, and 5 years are shown in Figure 8. It is important to note that 5-year and 1-year survival rate differences for recipients of living vs. deceased donors should be determined with caution, as the cohorts of living donor recipients have shifted from mostly pediatric to mostly adult. Until more adult-to-adult living transplants are made, direct comparison will be difficult.

As with graft survival, the youngest and oldest recipients had poorer patient survival rates among groups with sufficient sample size; all other age groups had similar patient survival rates. African American recipients exhibited reduced patient survival rates in most of the survival time cohorts reported. Cadaveric organ recipients with blood type $A B$ had improved survival rates in both short and long-term survival cohorts. Also paralleling the graft survival data, the most severely ill recipients had poorer patient survival rates for all cohorts. Candidates transplanted with cadaveric organs for cholestatic liver disease or biliary atresia had the best patient survival rates in the short and long-term (5-year survival rates of $80 \%$ and $86 \%$, respectively). It is interesting to note that recipients transplanted for malignant neoplasms had 5-year survival rates in excess of $50 \%$ - a respectable cancer survival statistic, especially because most of these recipients have hepatocellular carcinoma, for which there is no other curative modality. Similarly, recipients with noncholestatic liver disease, the majority of whom carry viral hepatitis, displayed an excellent 5-year survival rate (>70\%), even though the recurrence rate of hepatitis C for these patients is virtually $100 \%$ (19). There has been a concern whether the longer-term outcome of patients

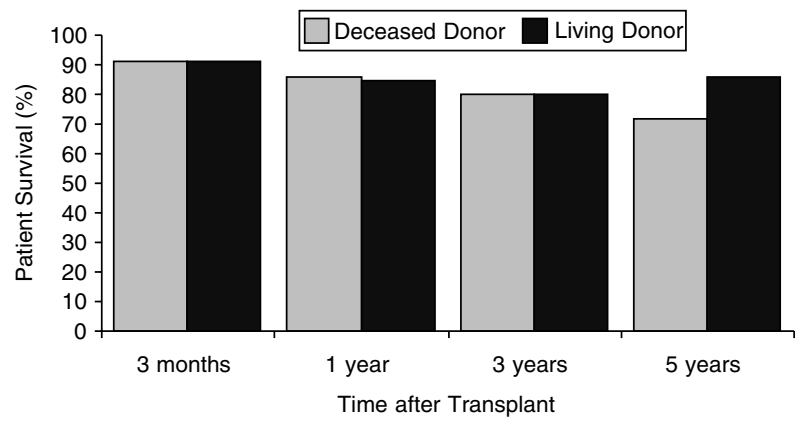

Figure 8: Patient survival among liver transplant recipients, living vs. deceased donor. Source: 2002 OPTN/SRTR Annual Report, Table 9.9. Cohorts are for transplants performed during 1999-2000 for 3-month and 1-year; 1997-1998 for 3-year; and 1995-1996 for 5 -year survival. with hepatitis $\mathrm{C}$ is worsening over time (17). The reasons for this trend are uncertain, but older deceased donor age may be an important factor (20). Three-month patient survival was slightly reduced at transplant centers performing fewer than 10 transplants per year, but survival rates were very similar for higher- and lower-volume centers at longer time periods after transplantation.

For recipients of livers from living donors, overall patient survival at 1 year was $85 \%$; for those receiving livers from deceased donors, overall patient survival at 1 year was $86 \%$. This was observed despite a smaller proportion of patients critically ill at time of transplantation undergoing living donor transplantation (Figure 8). In patients who were critically ill at time of transplantation (Status 1 or on life support), 1-year survival was $56-60 \%$ in living donor liver recipients, compared with $71-80 \%$ in deceased donor liver recipients.

\section{Model for end-stage liver disease}

Because of the many factors-increasing number of deaths on the liver waiting list, the inability to accurately categorize liver patients according to severity of liver disease using the partially subjective Child-Turcotte-Pugh classification, and reports suggesting that waiting time correlates poorly with death on the waiting list-a consensus opinion emerged that a revised allocation scheme was needed (4,21-23). The new liver allocation system implemented by the OPTN in February 2002 is based primarily on the severity of liver disease as assessed by the Model for End-stage Liver Disease (MELD) and Pediatric End-stage Liver Disease (PELD) survival models for all patients with chronic liver disease. The MELD score is based on three biochemical variables-serum bilirubin, serum creatinine, and international normalized ratio (INR) - and has been shown in retrospective and prospective studies to be highly predictive of 3-month mortality in patients with chronic liver disease. Similarly, the PELD model for pediatric patients was developed based on analyses of data from the Study of Pediatric Liver Transplantation (SPLIT) database and has been shown retrospectively to be predictive of waiting list mortality in pediatric patients (24). The MELD and PELD model equations appear in Table 1. In Figure 9, the relationship between MELD and PELD and mortality risk at 3 months is shown. Mortality risk was derived from 649 pediatric patients on the SPLIT database and 1230 adult patients added to the waiting list between March 1, 2001 and August 15, 2001. These regression models may change over time as more data are accumulated. In addition, other allowances may need to be made to avoid disadvantaging those patients with good indications for liver transplant but without significant synthetic liver dysfunction (5). Candidates with hepatocellular cancer (HCC) make up the largest group in this category. It is hoped that these models will allow for better counseling for patients regarding the probability of death while waiting for transplantation. This 


\section{Roberts et al.}

Table 1: MELD and PELD score equations

\begin{tabular}{|c|c|}
\hline Score type & Equation \\
\hline $\begin{array}{l}\text { MELD score } \\
\text { (Malinchoc et al. (25)) }\end{array}$ & $\begin{aligned} \mathrm{R}= & 0.957 \times \log _{\mathrm{e}}(\text { creatinine } \mathrm{mg} / \mathrm{dL})+0.378 \times \log _{\mathrm{e}}(\mathrm{bilirubin} \mathrm{mg} / \mathrm{dL}) \\
& +1.120 \times \log _{\mathrm{e}}(\mathrm{INR})+0.643 \times(\text { disease etiology })\end{aligned}$ \\
\hline MELD score, New Policy & $\begin{aligned} \mathrm{R}= & \left.\left(0.957 \times \log _{\mathrm{e}} \text { (creatinine } \mathrm{mg} / \mathrm{dL}\right)+0.378 \times \log _{\mathrm{e}} \text { [total bilirubin } \mathrm{mg} / \mathrm{dL}\right] \\
& \left.+1.120 \times \log _{\mathrm{e}}[\mathrm{INR}]+0.643\right) \times 10\end{aligned}$ \\
\hline PELD score & $\begin{aligned} \mathrm{R}= & \left(0.463\left[\text { age\#] }-0.678 \times \log _{\mathrm{e}} \text { [albumin } \mathrm{g} / \mathrm{dL}\right]+0.480 \times \log _{\mathrm{e}} \text { [total bilirubin } \mathrm{mg} / \mathrm{dL}\right] \\
& \left.\left.+1.857 \times \log _{\mathrm{e}}[\mathrm{INR}]+0.667 \text { [growth failure }{ }^{*}\right]\right) \times 10\end{aligned}$ \\
\hline
\end{tabular}

Note: MELD score equations as reported by Malinchoc et al. (25) and as incorporated in the new liver allocation policy. The disease etiology variable included in the Malinchoc et al. equation has been omitted but the constant is maintained. PELD score includes bilirubin, albumin, and INR continuous variables and dichotomous variables for age and growth failure.

*1 for noncholestatic disease, 0 for cholestatic disease.

$\#<1$ year of age $+1, \geq 1$ year of age $=0$.

** $>2$ standard deviations below the mean for age $=1, \leq 2$ standard deviations below the median for age $=0$.

is important when the patient is considering issues such as whether to undergo living donor transplantation. It is hoped that the time horizon for which the models can predict death could be extended from 3 months to possibly as long as a year. Recent reports have indicated that the MELD score is an accurate predictor of 1-year, pretransplant survival (21).

The new MELD/PELD system was activated on February 27,2002 . The trend toward fewer liver transplant waiting list registrations noted in the 6 months prior to implementation continued after February 27, 2002. The reasons for this decrease are unclear. For all patients listed as of February 27, 2002, plus those non-Status 1 candidates added through June 1, 2002, 93\% of adults and 93\% of pediatric patients listed for liver transplantation had a MELD/PELD score of less than 20 (Figures 10 and 11).

In adults listed between May 11, 2002 and July 31, 2002, there was minimal difference between mean MELD score at listing for males and females (15.0 vs. 15.6). African Americans had slightly higher mean MELD score at listing (17.5) compared with whites (15.2) and Asians (14.5). Patients listed for retransplantation had a higher mean MELD score (19.9) compared with those patients undergoing primary transplant (15.0). There was no difference in

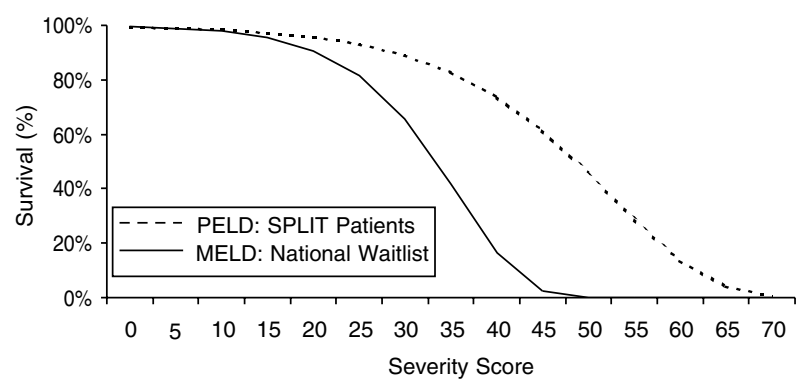

Figure 9: MELD and PELD mortality risks at 3 months for 1230 adult and 649 pediatric patients added to waiting list between 3/1/01 and 8/15/01. Source: Liver Transplantation 2002; 8: 854. the mean MELD score among ABO blood types. The mean MELD score at listing in the 11 OPTN/UNOS regions varied minimally, ranging from 14.6 to 16.3 in adult patients listed for liver transplantation.

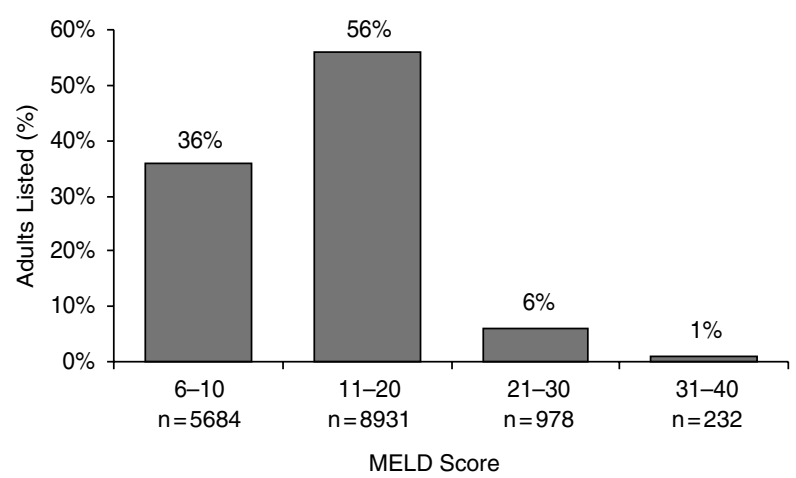

Figure 10: Adult registrants on liver waiting list by MELD score. Source: SRTR Data Analyses, 2002. Cohort includes nonexception liver candidates listed between 2/27/02 and 6/1/02.

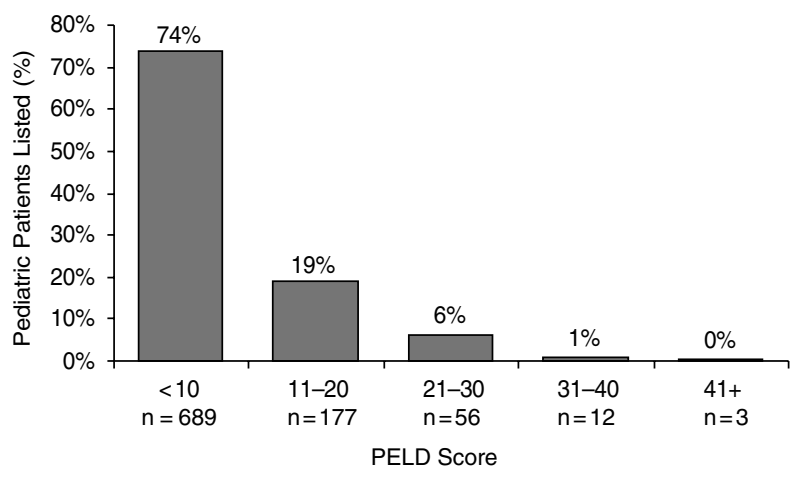

Figure 11: Pediatric registrants on liver waiting list by PELD score. Source: SRTR Data Analyses, August 2002. Cohort includes nonexception liver candidates listed between 2/27/02 and 6/1/02. 
Among pediatric patients (younger than 18) listed between May 11, 2002 and July 31, 2002, there was no difference in mean PELD scores by gender. The mean PELD score at listing was 10.3 for females and 11.6 for males. Similar to the adult results, the PELD scores for African Americans (13.5) were higher than the scores of whites (10.2) or Asians (12.0). Patients less than 1 year of age (who receive additional PELD points) still had a much higher mean PELD score (mean 16.2) at listing compared with a PELD score of 7.1 for children ages 1-10 years and a mean PELD score of 7.0 for children ages 11-17. Most of the patients younger than 1 year had biliary atresia, which is associated with higher severity of disease than other pediatric liver diagnoses. Unlike adults, pediatric patients listed for retransplantation had a median PELD score of 11.8, virtually identical to that of patients listed for primary transplant. In assessing the OPTN/UNOS regions, there was a wider range of mean PELD scores at listing compared with the adults. The mean PELD score at listing ranged from 7.1 to 12.7. However, two of the OPTN/ UNOS regions listed small numbers of pediatric patients (fewer than 20), which likely accounts for the wide variability.

A preliminary assessment of 30-day outcomes based on MELD/PELD scores was conducted among patients listed for liver transplantation on February 27, 2002 or who registered between February 28, 2002, and June 1, 2002. All patients had at least 30 days of follow-up from February 27, 2002 or the later date of listing. In addition, it should be noted that patients with hepatocellular cancer were divided into those who had stage T1 and those who had stage T2 disease. The new allocation policy stipulates that patients with stage T1 HCC are given a MELD/PELD score of 24 and patients with stage T2 HCC can qualify for a MELD/PELD score of 29 , if they meet specific criteria, regardless of their laboratory values. These scores, corresponding to $15 \%$ and $30 \%$ probabilities of dying within 3 months, respectively, were derived from initial estimates of the risk of progression beyond stage T2 disease.

Figures 12 and 13 show the percentage of candidates who were transplanted within 30 days of listing, based on their MELD/PELD scores, excluding candidates with hepatocellular cancer. Similarly, Figures 14 and 15 show the percentages of candidates dying on the waiting list within 30 days of listing. These data indicate that approximately $38 \%$ of candidates with a MELD/PELD score greater than 30 were transplanted within 30 days. However, the percentage of adults with MELD scores greater than $30(22 \%)$ who died while waiting was greater than the percentage of pediatric patients with PELD scores greater than $30(17 \%)$ who died while waiting. This is expected because the MELD score of 30 represents a 3-month mortality risk of $33 \%$ compared with a PELD score of 30 , indicating a 3-month mortality risk of $19 \%$ in pediatric patients. Pediatric patients have a higher death rate at lower scores of $11-20$. The lower mortality rate for pediatric

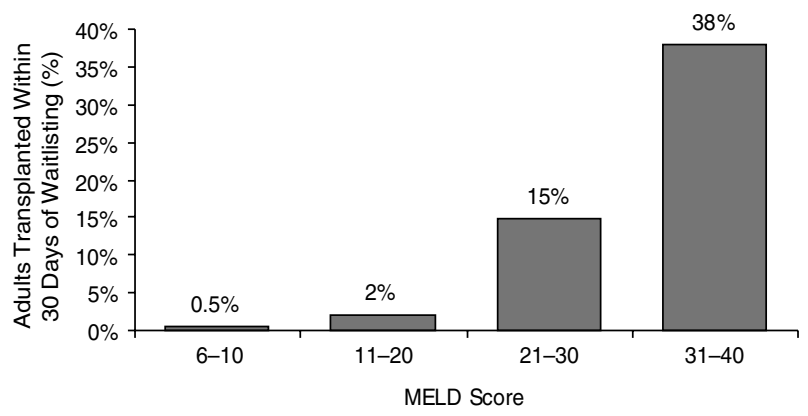

Figure 12: Percentage of adult registrants on liver waiting list transplanted within 30 days of listing by MELD score. Source: SRTR Data Analyses, August 2002. Cohort includes nonexception liver candidates listed between 2/27/02 and 6/1/02.

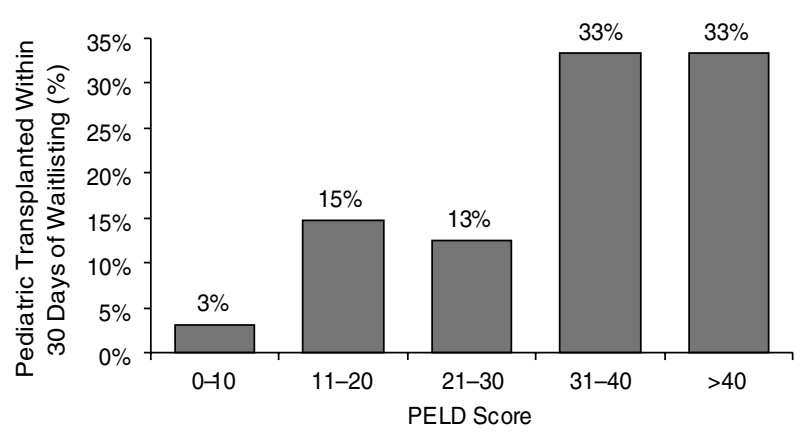

Figure 13: Percentage of pediatric registrants on liver waiting list transplanted within 30 days of listing by PELD score. Source: SRTR Data Analyses, August 2002. Cohort includes nonexception liver candidates listed between 2/27/02 and 6/1/02.

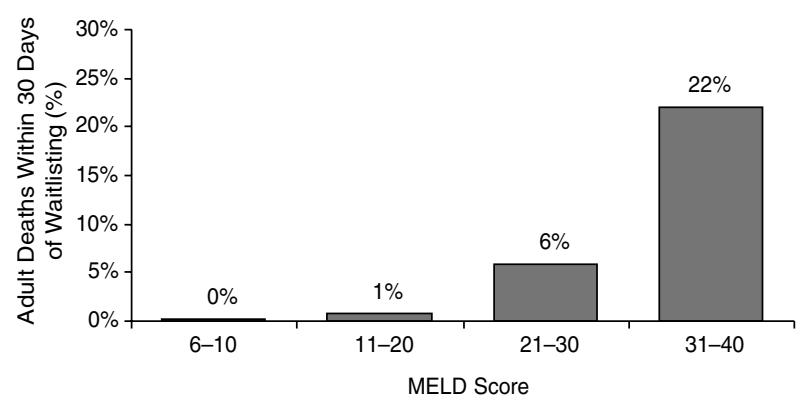

Figure 14: Percentage of adult deaths on liver waiting list within 30 days of listing by MELD score. Source: SRTR Data Analyses, August 2002. Cohort includes nonexception liver candidates listed between 2/27/02 and 6/1/02.

patients at higher PELD scores may be because pediatric patients with chronic liver disease who require intensive care have the option to be made Status 1 in order to avoid death-an option not available to adult patients. It is therefore difficult to interpret the ability of PELD to predict death, especially at higher scores. 


\section{Roberts et al.}

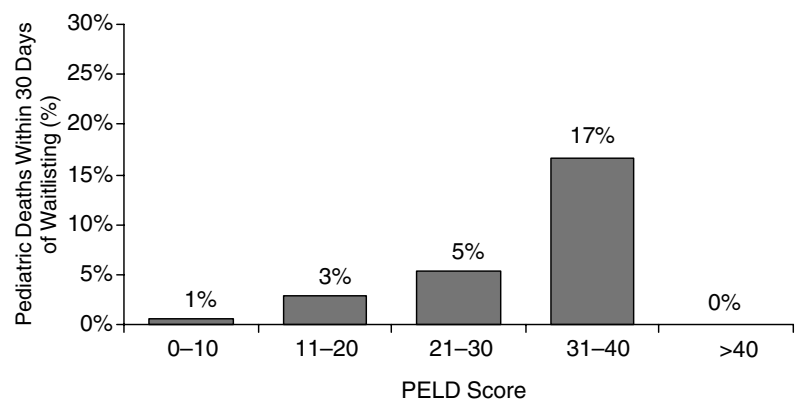

Figure 15: Percentage of pediatric deaths on liver waiting list within 30 days of listing by PELD score. Source: SRTR Data Analyses, August 2002. Cohort includes nonexception liver candidates listed between 2/27/02 and 6/1/02.

It should be noted that the risk of death at the lower MELD scores observed for patients on the waiting list is lower than the risks of death predicted by the original version of the formula (25). This may be due to the fact that the mortality risks for patients prioritized for transplant by the MELD/PELD system is reduced because they receive a transplant (rather than dying) compared with cohorts of patients not prioritized for transplant on whom the original MELD/PELD equations were derived and validated.

The median lab MELD score for all nonexception adults at the time of transplant across the 11 OPTN/UNOS regions ranged from 16 to 28. Pediatric recipients exhibited a considerably lower range, 0-22 (as reported by the SRTR). The wide range of PELD scores for pediatric patients reflects the small number of patients reported as transplants in several regions. Furthermore, an initial analysis of patients with hepatocellular cancer undergoing liver transplant based on staging revealed that $25 \%$ of patients with T1 HCC (MELD score of 24) were transplanted within 30 days and $43 \%$ of patients with T2 HCC (MELD score 29) were transplanted within 30 days. In this group of the study, few patients died within the 30-day follow-up. The mean calculated MELD score for patients with hepatocellular cancer was 11.6 for patients having T1 disease and 12.7 in patients having T2 disease.

While some of the preliminary analyses of the MELD/ PELD data are intriguing, other important questions must await further data accrual. Some questions must be further addressed: What is the impact of the MELD/ PELD system on the incidence of deaths on the waiting list? Has the prorated MELD score for patients with hepatocellular cancer had a detrimental effect on patients with chronic liver disease without hepatocellular cancer? How accurate is our assessment of hepatocellular cancer with regard to diagnosis, size of tumor, and number of tumors? What impact will the MELD/PELD system have on overall resource utilization and on patient and graft survival in patients undergoing liver transplantation? Are there marked variations at the regional and organ procurement organization (OPO) levels in the MELD/PELD scores of patients undergoing transplantation (suggesting inequities in the allocation system)? It is hoped that the answers to many of these questions will emerge in the coming year.

\section{Intestine Transplantation}

In contrast to liver allocation policy, there have been no substantial changes to the allocation of intestines between 1993 and 2001. Although the current demand for intestinal transplants is relatively modest (180 patients waiting at the end of 2001), this is an increase of over $300 \%$ since 1993 , the first year of the intestine waiting list. In 2001, 111 intestinal transplants were performed, and short gut syndrome was the reported reason for more than $60 \%$ of these transplants. Children younger than 6 years were the recipients of almost half of all intestine transplants in 2001. In 2001, the median time to transplant for pediatric patients ranged from 173 days to 488 days. The comparable range for adults was 46 days to 129 days. Remarkably, death rates for wait-listed intestine transplant candidates tend to be substantially higher than those of wait-listed liver candidates, particularly among candidates under 6 years of age. In 2001, the overall death rate among intestine candidates was 272 per 1000 patient years compared with a rate of 115 for liver candidates. As with other organs, the number of deceased donors has not kept pace with the number of intestinal candidates. Waiting list data for 1993-99 shows that since 1994, the death rate for patients on total parenteral nutrition with a diagnosis of short gut syndrome who develop liver failure has greatly exceeded that for all other candidates waiting for livers. While the overall liver transplant waiting list death rate has steadily decreased, it has increased sixfold in the short gut/parenteral nutrition patient subset (26). The majority of intestinal transplants have been performed in only four transplant centers in the country. Outcomes following intestinal transplantation have been less impressive than those following liver transplantation. The overall 1-year and 5-year graft survival rates were 66\% and $20 \%$; corresponding graft survival rates for liver transplants were $80 \%$ and $64 \%$. Graft survival rates following intestinal transplantation were better for recipients who were adults, who had blood type $\mathrm{O}$ or $\mathrm{A}$, or who had not had a previous intestine transplant.

The current literature does not indicate a marked difference in outcomes between recipients of liver/intestinal grafts vs. those of isolated intestinal grafts. This is supported by pooled data from the International Intestinal Transplant Registry (ITR) (27), as well as center-specific data (28-30). Although data do exist to substantiate an early survival advantage in recipients of isolated intestinal grafts (31), the long-term graft outcomes are better for the combined liver-intestinal grafts, although the difference is not statistically significant (27-30). Recent improvements 
in outcome may be attributed to tacrolimus-based immunosuppression strategies and newly developed adjunct agents, including interleukin-2 receptor antagonists and rapamycin (32-35).

\section{Intestine waiting list characteristics}

The total number of patients listed for intestinal transplantation has continued to grow each year and reached 180 in 2001. As noted above, this rise represents an increase of more than $300 \%$ from 1993. The patient demographics indicate that the majority (approximately $75 \%$ ) of patients are under 18 years of age. The majority of patients are white (76\%), non-Hispanic/non-Latino (81\%), blood type O $(44 \%)$, residents of the United States (94\%), and have not had a previous transplant (91\%). The gender distribution is nearly even. Approximately $25 \%$ of patients on the waiting list at the end of the year 2001 had been waiting less than 3 months for their transplant, 25\% 3 to $<12$ months, and 50\% 1 year or longer. This distribution represents a shift toward longer waiting times.

\section{Intestine time to transplant}

There were 219 new registrations for intestine transplants in 2001. The median time to transplant for all patients was 319 days. Age appears to influence waiting time: Candidates younger than 1 year waited 208 days (25th percentile) to be transplanted, and candidates ages 11-17 years waited 337 days; all other age groups for which data were available waited less than half that time. Data on the median time to transplant by race is limited because of small patient numbers. White registrants had a slightly shorter waiting time (278days) than African American registrants (333 days); no substantial data were available for other racial groups. Analysis of ethnicity revealed a similar trend in median time to transplant: Hispanic/Latino registrants waited less (185 days) than non-Hispanic/nonLatino registrants (333 days); registrants of unknown ethnicity waited 208 days. Males had a longer median waiting time than females, 337 days compared with 242 days. A similar trend was seen when the analysis was performed by $A B O$ blood group: 333, 194, and 337 days, respectively, for blood types $\mathrm{O}, \mathrm{A}$, and $\mathrm{B}$. Patients with a prior history of transplantation also had a slightly longer 25th percentile time to transplant ( 105 days) than those without a history of previous transplantation (81 days). US residents had a median time to transplantation of 319 days, and the nonresident group had a shorter time of 101 days.

\section{Intestine waiting list death rates}

In 2001, there were a total of 349 registrants for intestine transplants at risk. The number of deaths during this year was 45, yielding a death rate of 272 deaths per 1000 patient years. Age had a substantial impact on death rates. The younger age groups (younger than 1 year and $1-5$ years) had death rates markedly higher than that of the total death rate (1116 and 367, respectively). All other age groups for which data were available had death rates lower than the overall death rate. Race, ethnicity, and gender had a limited effect on death rates. Analysis of death rate by blood type revealed recipients with blood type $O$ had a death rate of 313 , while those with blood type $A$ had a higher death rate (324) and those with blood type B had a lower death rate (159).

The waiting list death rates in this group of patients are substantially higher than those reported for any other solid organ transplant candidate group. Pretransplant mortality rates as high as 50\% have been reported in the literature from individual transplant centers $(31,36)$. While the etiology of this mortality is speculative, the advanced medical condition of these patients at the time of evaluation and the limited availability of suitably sized and quality donors no doubt contributes significantly. It remains to be seen if the institution of the MELD/PELD scoring system for liver allocation will help reduce these mortality figures, as this scoring system was analyzed in patient populations that excluded candidates for liver/intestinal transplantation.

\section{Intestine transplant recipient characteristics}

In 2001, 111 patients underwent transplantation involving the intestine-the most of any year of the registry. The age distribution for these recipients is bimodal, with the majority $(48 \%)$ younger than 6 years old and the second most common age group (32\%) aged 35-64 years. Short gut syndrome was the most common primary diagnosis. Examination of the International Intestinal Transplant Registry data reveals that the leading indications for intestinal transplant in adults are ischemia, Crohn's disease, trauma, and tumor, while those for children are volvulus, gastroschisis, necrotizing enterocolitis, and pseudoobstruction (27).

Interestingly, $62 \%$ of the recipients in the OPTN/SRTR data were not hospitalized at the time of transplant in 2001. This represents a substantial change over time. In 1993, 59\% of recipients were either hospitalized or in intensive care at time of transplant (Figure 16). However, the percentage of patients on life support at time of transplant did not change substantially between 1993 (6\%) and 2001 (4\%).

\section{Intestine transplantation post-transplant death rates}

For the year 2001, there were a total of 138 patients at risk, 26 deaths, and a death rate per 1000 patient years of 442. This death rate is an increase over the death rates for 1999 (412) and 2000 (196), returning to levels similar to those before 1999 .

Analysis of the death rate by recipient age at transplant reveals several interesting trends. First, while few data exist for patients younger than 1 year, the death rate in the next group, ages 1-5years (684), is substantially higher than all other age groups. Other publications 
Roberts et al.

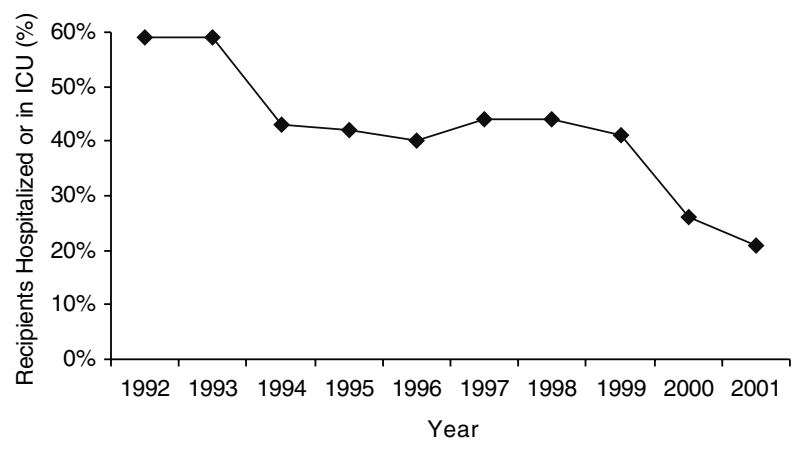

Figure 16: Percentage of intestine transplant recipients hospitalized or in intensive care unit at transplant, 1992-2001. Source: 2002 OPTN/SRTR Annual Report, Table 10.4.

indicate that the death rate after intestinal transplantation in very young children is very high, corroborating this trend (37). All other age groups have death rates lower than that of the total.

Insufficient patient numbers for 2001 limit death rate analysis for the racial groups Asian and Other/Multi-race categories. However, the death rate of white recipients was slightly less (361) than that of the total group (442), while the death rate of African American recipients was substantially higher (905) than that of the total group. Regarding ethnicity, Hispanic/Latino recipients had the lowest death rate (245), while the non-Hispanic/non-Latino and Unknown recipients had death rates equal to or greater than that of the overall group. Males had a higher death rate (472) than did females (406). Blood type appeared to have a modest influence on death rate. Blood types $\mathrm{O}$ and $B$ had lower death rates than the overall group, while recipients of blood type $A$ had a higher death rate (756) than the overall group. US residents had a death rate of 395, nonresident aliens had no deaths during 2001, and those of unknown residency had a death rate of 1286. A history of previous organ transplant markedly increased the post-transplant death rate $(1152$, compared with 385 for first-time recipients).

The pretransplant status of the patient appears to affect outcomes after intestinal transplantation. Patients at home before transplantation had death rates lower than that of the total group. Patients hospitalized but not in intensive care had a death rate (288) much lower than that of the total group. Unfortunately, analyses based upon intensive care, as well, as the variable life support status, were limited because of small patient numbers.

The primary diagnosis of the recipient did not appear to influence the death rate. Donor age, however, appeared to affect the death rate. Recipients of intestines from donors with ages younger than 1 year had a death rate of 795 per 1000 patient years at risk. Recipients of donors of all other ages had death rates equal to or less than that of the total group death rate.

Cause of death is an important variable for interpretation of outcomes. By far, the most common cause of death reported in the literature after intestinal transplantation is infection and multisystem organ failure $(27-30,38)$. These recipients appear to be very susceptible to infectious complications and death because of their frequent history of multiple pretransplant infections, inclusion of an intestinal graft with its obligate endogenous microflora, and requirement for high dose immunotherapy after transplantation.

\section{Intestine transplantation graft survival}

There were no consistent patterns for graft survival among recipients in different age groups. Those recipients aged $6-10$ years had a particularly high 3-year graft survival (80\%). Recipients aged 11-17 years demonstrated a longterm survival similar to the youngest age groups.

Very few nonwhites have received intestinal allografts, which makes comparisons of graft survival by race unfeasible. Analysis by ethnicity tended to show better short-term results among Hispanic/Latino recipients. Male recipients had higher graft survival than females at 3 months $(83 \%$ vs. $70 \%)$, 1 year $(71 \%$ vs. $61 \%)$, and 3 years $(55 \%$ vs. $31 \%)$ but worse 5 -year graft survival $(12 \%$ vs. $28 \%)$. For unknown reasons, recipients with blood type B had a markedly lower graft survival than those with other blood types at all time points. Prior history of intestinal transplant also negatively influenced graft survival (43\% compared with $70 \%$ for those without prior history at 1 year post-transplant).

Patients not hospitalized before intestinal transplantation had higher graft survival (16-88\%) at all intervals than those hospitalized or in an ICU (0-47\%). Patients hospitalized before transplantation demonstrated the lowest graft survival at all intervals (0-27\%). Those in intensive care before transplantation had early graft survival rates higher than the hospitalized group, but this advantage was lost after 3 years. The need for life support before transplantation also was associated with a lower graft survival rate (0\% compared with 69\% for those not on life support at 1 year post-transplant). Primary diagnosis appeared to have no effect.

Transplant center volume did not appear to influence graft survival during the early interval. Small (0-1 transplants per year), medium (2-7 transplants per year), and large (8 or more transplants per year) demonstrated comparable 3-month and 3-year survival. However, the medium-sized programs demonstrated the best 5-year survival (35\%). These data are in contrast to the ITR data from 1997, which indicated that large centers (more than 10 transplants per year) had a significantly better outcome than those centers performing fewer than 10 transplants per year (27). 


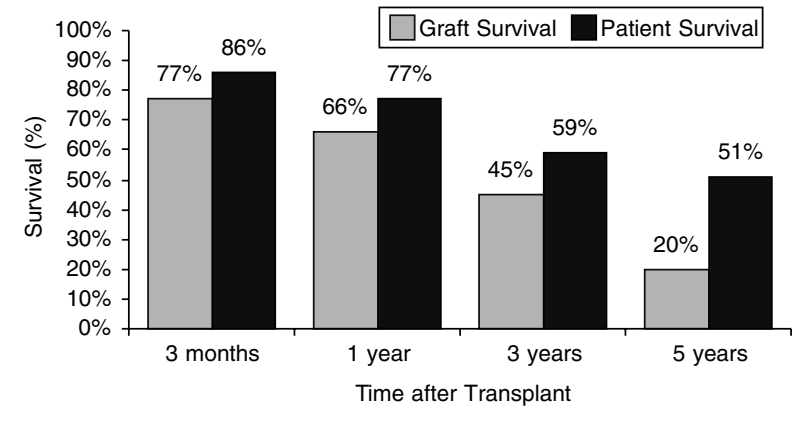

Figure 17: Graft vs. patient survival among intestine transplant recipients. Source: 2002 OPTN/SRTR Annual Report, Tables 10.8, 10.9. Cohorts are for transplants performed during 1999-2000 for 3-month and 1-year; 1997-1998 for 3-year; and 1995-1996 for 5 -year survival.

Graft survival analyzed by donor age revealed that recipients of grafts from donors younger than 1 year fared the worst during the 3-month and 1-year time intervals $(50 \%$ and $17 \%$, respectively). Recipients of grafts from donors aged $35-49$ years had the best 3-year survival $(100 \%)$, but small sample size makes the comparison of age groups difficult. However, 5-year graft survival was nearly equal in all groups with data available.

Cause of graft loss is important. Published series overwhelmingly indicate that the major cause of graft loss after intestinal transplantation is immunologic due to acute or chronic rejection $(27-30,38)$. Overall graft and patient survival for intestine transplant recipients for 3 months, 1 year, 3 years, and 5 years are shown in Figure 17.

\section{Intestine transplantation patient survival}

The overall rates of patient survival following intestine transplantation were $86 \%$ after 3 months, $77 \%$ after 1 year, $59 \%$ after 3 years, and $51 \%$ after 5 years. Analysis of patient survival by recipient age revealed that there were no major differences in 3-month or 5-year patient survival in any subgroup with sufficient sample size. However, patient survival at 1 year following transplant was lower for recipients under 1 year old (53\%). Among those groups with sufficient sample size, patient survival rates at 3 years were lowest for those under 1 year old $(50 \%)$ and those aged $35-49$ years $(46 \%)$.

African American recipients had lower 1-year and 3-year survival rates (64\% and $47 \%$, respectively) than white recipients. African American recipients in the 1995-1996 cohort fared much better (73\%) than whites in the same cohort at 5-year patient survival. Ethnicity only appeared to influence patient survival at the 3-year mark, when Hispanic/Latino recipients demonstrated a much better patient survival (92\%) than non-Hispanic/non-Latino recipients (56\%). Recipients with blood type B demonstrated a lower survival rate at 3 months and 1 year post-transplant $(60 \%)$ than those with other blood types.
Patients hospitalized or in intensive care before transplant had a significantly lower 1 -year survival $61 \%$ and $46 \%$, respectively) than those not hospitalized before transplant $(84 \%)$. At 3 years following transplantation, patient survival was markedly lower for patients who had been in an ICU before transplantation (21\%). By 5 years, both of these differences were gone. Patients with short gut syndrome demonstrated a lower 3-year survival (54\%) than those with functional bowel problems $(71 \%)$. Those who received intestine transplants at small centers appeared to have a lower 5 -year survival rate $(46 \%)$. Donor age had little effect on patient survival, though recipients of intestines from donors aged 18-34 years had a higher 1-year survival rate $(91 \%)$ than those with younger or older donors.

\section{Conclusions}

Liver transplantation is the second most common transplant ( $21 \%$ of all organ transplants), while intestine transplants occur only rarely. The biggest development in liver transplantation in the United States over the last several years has been the rapid increase in the number of living donor transplants. Although this procedure accounts for only $10 \%$ of the liver transplants performed in 2001, the number has more than doubled since 1999. Early graft survival after living donor transplantation appears to be lower than that following cadaveric transplantation, but long-term graft survival is superior. It is hoped that the additional benefit of reduced waiting list mortality from earlier transplantation, along with continued maturation of this new surgical procedure, will offer benefit to patients awaiting liver transplantation. The Model for End-stage Liver Disease (MELD) and the Pediatric End-stage Liver Disease (PELD) model were instituted for a refined prioritization of patients with chronic liver disease awaiting transplantation. Despite the very recent institution of the new allocation policy (February 2002), some preliminary analyses are available and indicate the questions that have yet to be addressed.

\section{References}

1. Trotter JF, Wachs M, Everson GT, Kam I. Medical progress. Adult-to-adult transplantation of the right hepatic lobe from a living donor. N Engl J Med 2002; 346: 1074-1082.

2. Dickinson DM, Ellison MD, Webb RL. Data sources and structure. Am J Transplant 2003; 3(Suppl. 4): 13-28.

3. Wolfe RA, Webb RL, Dickinson DM et al. Analytical approaches for transplant research. Am J Transplant 2003; 3(Suppl. 4): 103-113.

4. Freeman RB Jr, Edward EB. Liver transplant waiting time does not correlate with waiting list mortality: Implications for liver allocation policy. Liver Transplant 2000; 6: 543-552.

5. Freeman RB Jr, Weisner RH, Harper $A$, et al. The new liver allocation system: Moving toward evidence-based transplantation policy. Liver Transplant 2002; 8: 851-858.

6. Freeman RB Jr, Rohrer RJ, Katz E, et al. Preliminary results of a liver allocation plan using a continuous medical severity score that de-emphasizes waiting time. Liver Transplant 2001; 7: 173-178. 


\section{Roberts et al.}

7. LaPointe Rudow D, Lee A, Hafliger S, Emond JC, Brown RS Jr. A cohort study of patients listed for liver transplant with and without living donors. Am J Transplant 2002; 2 (Suppl 3): 236.

8. Testa G, Malago $M$, Nadalin $S$ et al. Right-liver living donor transplantation for decompensated end-stage liver disease. Liver Transplant 2002; 8: 340-346.

9. Kam I. Editorial: Adult-adult right hepatic lobe living donor liver transplantation for status 2a patients: Too little, too late. Liver Transplant 2002; 8: 347-349.

10. Surman OS. The ethics of partial-liver donation. N Engl J Med 2002; 346: 1038.

11. Nair S, Eustace J, Thuluvath PJ. Effect of race on outcome of orthotopic liver transplantation: a cohort study. Lancet 2002; 359: 287-293.

12. Pruthi J, Medkiff KA, Esrason KT et al. Analysis of causes of death in liver transplant recipients who survived more than 3 years. Liver Transplant 2001; 7: 805-811.

13. Rabkin JM, de La Melena V, Orloff SL, Corless CL, Rosen HR, Olyaei AJ. Late mortality after orthotopic liver transplantation. Am J Surg 2001; 181: 475-479.

14. Roayaie S, Frischer JS, Emre SH et al. Long-term results with multimodal adjuvant therapy and liver transplantation for the treatment of hepatocellular carcinomas larger than 5 centimeters. Ann Surg 2002; 235: 533-539.

15. Verran DJ, Gurkan A, Dilworth $P$ et al. Inferior liver allograft survival from cadaveric donors $>50$ years of age? Clin Transplant 2001; 15: 106-110.

16. Oh CK, Sanfey HA, Pelletier SJ, Sawyer RG, McCullough CS, Pruett TL. Implication of advanced donor age on the outcome of liver transplantation. Clin Transplant 2000; 14: 386-390.

17. Berenguer $\mathrm{M}$, Prieto $\mathrm{M}$, San Juan F et al. Contribution of donor age to the recent decrease in patient survival among HCV-infected liver transplant recipients. Hepatology 2002; 36: 202-210.

18. Edwards EB, Roberts JP, McBride MA, Schulak JA, Hunsicker LG. The effect of the volume of procedures at transplantation centers on mortality after liver transplantation. N Engl J Med 1999; 341: 2049-2053.

19. Berenguer M, Lopez-Labrador FX, Wright TL. Hepatitis C and liver transplantation. J Hepatol 2001; 35: 666-678.

20. Wali M, Harrison RF, Gow PJ, Mutimer D. Advancing donor liver age and rapid fibrosis progression following transplantation for hepatitis C. Gut 2002; 51: 248-252.

21. Wiesner RH, McDiarmid SV, Kamath PS et al. MELD and PELD. Application of survival models on liver allocation. Liver Transplant 2001; 7: 567-580.

22. Kamath PS, Wiesner RH, Malinchoc M et al. A model to predict survival in patients with end-stage liver disease. Hepatology 2001; 33: 464.

23. Committee on Organ Procurement and Transplantation Policy. Organ Procurement and Transplantation. Assessing current policies and potential impact of the DHHS Final Rule. Committee on Organ Procurement and Transplantation Policy, Division of Health Sciences Policy, Institute of Medicine. Washington, DC: National Academy Press, 1999: 75.

24. McDiarmid SV, Anand R, Lindblad AS et al. Development of a pediatric end-stage liver disease score to predict poor outcome in children awaiting liver transplantation. Transplantation 2002; 74: 173-181.

25. Malinchoc M, Kamath PS, Gordon FD et al. A model to predict poor survival in patients undergoing transjugular intrahepatic portosystemic shunts. Hepatology 2000; 31: 864-871.

26. Fryer JP, Pellar S, Ormond D. Inappropriate prioritization of patients needing liver/intestine transplants leads to very high waiting list mortality. Abstracts of the 2002 American Transplant Congress, April 27-May 1, 2002, Washington, DC, 2002.

27. Grant D. Intestinal transplantation. 1997 Report of the International Registry. Transplantation 1999; 67: 1061-1064.

28. Abu-Elmagd $\mathrm{K}$, Reyes J, Bond $\mathrm{G}$ et al. Clinical intestinal transplantation. A decade of experience at a single center. Ann Surg 2001; 234: 404-417.

29. Nishida S, Levi D, Kato $T$ et al. Ninety-five cases of intestinal transplantation at the University of Miami. J Gastrointest Surg 2002; 6: 233-239.

30. Langnas A, Chinnakotla S, Sudan D et al. Intestinal transplantation at the University Nebraska Med Center: 1990-2001. Transplant Proc 2002; 34: 958-960.

31. Sudan D, Kaufman SS, Shaw BW Jr et al. Isolated intestinal transplantation for intestinal failure. Am J Gastroenterol 2000; 95: 1506-1515.

32. Bond GJ, Reyes J, Mazariegos GV, Sindhi R, Abu-Elmagd K. Intestinal transplantation: a decade's experience at one institution. Abstracts of the 2nd International Congress on Immunosuppression. December 6-8, 2001. San Diego: 2001.

33. Sudan $D$, Chinnokotla $S$, Horslen $S$ et al. Basiliximab decreases the incidence of acute rejection after intestinal transplantation. Transplant Proc 2002; 34: 940-941.

34. Abu-Elmagd K, Fung J, McGhee $W$ et al. The efficacy of daclizumab for intestinal transplantation: Preliminary report. Transplant Proc 2000; 32: 1195-1196.

35. Fishbein TM, Florman S, Gondolesi G et al. Intestinal transplantation before and after the introduction of sirolimus. Transplantation 2002; 73: 1538-1542.

36. Beath SV, Protheroe SP, Brook GA et al. Early experience of pediatric intestinal transplantation in the United Kingdom, 1993 to 1999. Transplant Proc 2000; 32: 1225.

37. Reyes J, Bueno J, Kocochis S et al. Current status of intestinal transplantation in children. J Pediatr Surg 1998; 33: 243-254.

38. Farmer DG, McDiarmid SV, Yersiz $\mathrm{H}$ et al. Outcome after intestinal transplantation: Results from one center's 9-year experience. Arch Surg 2001; 136: 1027-1032. 